\title{
ON CRITERIA OF KASAHARA AND BLUMENTHAL FOR INNER-PRODUCT SPACES ${ }^{1}$
}

\author{
MAHLON M. DAY
}

1. Historical remarks. It is the purpose of this paper to give related generalizations of two quite distinct recent generalizations of the Jordan-vonNeumann characterization of inner-product spaces. $\mathrm{Kasahara}^{2}$ [6] gave a condition characterizing inner-product spaces among normed linear spaces. In [1] and [2] Blumenthal gave conditions characterizing inner-product spaces among complete, metrically convex, externally convex, metric spaces. ${ }^{3}$

The classical result of Jordan and von Neumann [4] says that $a$ normed linear space $E$ has a norm determined from a positive-definite inner product $\left(\right.$, ) by the rule $\|x\|^{2}=(x, x)$ if and only if the norm in $E$ satisfies the following condition:

$$
\begin{gathered}
\text { Each pair } x, y \text { of points of } E \text { satisfies the equation } \\
2\left(\|x\|^{2}+\|y\|^{2}\right)=\|x-y\|^{2}+\|x+y\|^{2}
\end{gathered}
$$

This result has been extended in different directions by different people. Day [3] put on the extra restriction that $\|x\|=\|y\|=1$ to get a weaker condition identifying inner-product spaces among normed linear spaces.

Each pair $x, y$ of points of $E$ such that $\|x\|=\|y\|=1$ satisfies

$$
4=\|x+y\|^{2}+\|x-y\|^{2} \text {. }
$$

In metric, but not necessarily linear, spaces let us write $p q$ for the distance between two points $p$ and $q$. Schoenberg [7] showed that among normed, or even semi-normed, linear spaces inner-product spaces are characterized by the ptolmaic inequality:

Presented to the Society, November 26, 1955 under the title Some criteria for inner product spaces; received by the editors September 24, 1958.

1 Author's note, added October 7, 1958. The original manuscript of this paper was submitted in October 1955 to Professor A. C. Schaeffer, then a member of the editorial committee of these Proceedings. The present version, except for this footnote, is identical with the original one. The unusually long time between submission and publication was caused by Professor Schaeffer's sudden death. During that time there appeared a paper by W. L. Stamey (Pacific J. Math. vol. 7 (1957) p. 1505), which adapted the queasy four-point property (used in this paper first and described in the author's Abstract 62-1-151 in the Bulletin of the Amer. Math. Soc.) to spherical and hyperbolic space.

2 Numbers in brackets refer to the bibliography at the end of this paper.

3 These terms are defined, for example, in [1]; for the convenience of the reader the definitions have been repeated in $\$ 3$ of this paper. 


$$
\begin{gathered}
\text { Each quadruple } p, q, r, s \text { of points of } E \text { satisfies } \\
\qquad p q \cdot r s+p r \cdot q s \geqq p s \cdot q r .
\end{gathered}
$$

Schoenberg also showed that either of the conditions $(S, \geqq)$ or $(S, \leqq)$ obtained from (D) by replacing equality by the relation $\geqq$ or the relation $\leqq$, also suffices to characterize inner-product spaces among linear normed spaces.

Kasahara [6] studies the normed case but weakens ( $\mathrm{JN}$ ) to:

There is a positive number $a \leqq 1 / 2$ and for each pair $x, y$ of

points of $E$ there is a number $\lambda$, depending on $x$ and $y$, such

$$
\lambda\|x\|^{2}+(1-\lambda)\|y\|^{2} \geqq \lambda(1-\lambda)\|x-y\|^{2}+\|\lambda x+(1-\lambda) y\|^{2} .
$$

Kasahara showed that his condition implies that a normed linear space $E$ has an inner product by showing that it implies Kakutani's condition [5];

(Kk) There is a projection of norm one on every closed linear subspace of $E$.

In purely metric terms W. A. Wilson [8] characterized subsets of inner-product spaces by use of the (0th) euclidean four-point property:

(e4pp) Each quadruple $p, q, r, s$ of points of $E$ is isometrically embeddable in a (three dimensional) euclidean space.

Blumenthal, [1] and [2], defines the first (weak) and second (feeble) euclidean four-point properties:

Each quadruple $p, q, r, s$ of points $E$ such that $\begin{array}{ll}\text { (e4pp-1) }[(\mathrm{e} 4 \mathrm{pp}-2)] & \begin{array}{l}p q+q r=p r[\text { and also } p q=q r] \text { is isometri- } \\ \text { cally embeddable in a (two-dimensional) eu- }\end{array}\end{array}$ clidean space.

In [1], pages 123-128, Blumenthal shows that the (e4pp-1) characterizes inner-product spaces in the class $S M$ of complete metrically convex, externally convex, semi-metric spaces. ${ }^{3}$ In [2] he shows that in the subclass $M$ of all metric spaces in $S M$ the condition (e4pp-2) implies (e4pp-1), and, therefore, characterizes inner-product spaces in the class $M$. He asserts that this is a purely metric generalization of the Jordan-von Neumann theorem, which in a sense is true. (See §3.)

In $\$ 2$ of this note I use Schoenberg's shrewd observations on the generality of my methods in [3] to give a common generalization of my result (D) and Kasahara's result (K); in particular, the uniformity needed by Kasahara is unnecessary by this method. In $\$ 3$ I give 
a result which generalizes Blumenthal's and also the condition $(1,=)$ of $\$ 2$ which is somewhat more restrictive than the generalization $(1, \geqq)$ of $(K)$.

2. Linear normed spaces. One elementary way to weaken Kasahara's condition is to drop the uniformity imposed by the existence of the number $a$. Also it is possible to consider the related criteria with $\geqq$ replaced by $=$ or $\leqq$ to get the conditions $(1, \sim)$ below, where $\sim$ stands for any given (but the same throughout $E$ ) one of $=$, $\geqq$ or $\leqq$.

For each pair $x, y$ of points of $E$ there is a number $\lambda$, depend$(1, \sim)$ ing on $x$ and $y$, such that $0<\lambda<1$ and

$$
\begin{aligned}
\lambda\|x\|^{2}+(1-\lambda)\|y\|^{2} \sim \lambda(1-\lambda)\|x-y\|^{2} & \\
& +\|\lambda x+(1-\lambda) y\|^{2} .
\end{aligned}
$$

Another extension comes from weakening these as (D) is formed from $(\mathrm{JN})$.

For each pair $x, y$ of points of $E$ with $\|x\|=\|y\|=1$ there $(2, \sim)$ exists a number $\lambda$, depending on $x$ and $y$, such that $0<\lambda<1$

$$
1 \sim \lambda(1-\lambda)\|x-y\|^{2}+\|\lambda x+(1-\lambda) y\|^{2} .
$$

To show that any one of these conditions is sufficient that $E$ be an inner-product space, let us begin by constructing a still more general property of inner-product spaces. If $E$ is an inner-product space, then for each pair $x, y$ of points of $E$ and each pair $\lambda, \mu$ of real numbers we have

$$
\begin{aligned}
\|\lambda x+(1-\lambda) y\|^{2} & =\lambda^{2}\|x\|^{2}+2 \lambda(1-\lambda)(x, y)+(1-\lambda)^{2}\|y\|^{2}, \\
\|\mu x+(1-\mu)(-y)\|^{2} & =\mu^{2}\|x\|^{2}-2 \mu(1-\mu)(x, y)+(1-\mu)^{2}\|y\| .
\end{aligned}
$$

Eliminating $(x, y)$ gives

$$
\begin{aligned}
\mu(1-\mu) \| \lambda x+ & (1-\lambda) y\left\|^{2}+\lambda(1-\lambda)\right\| \mu x-(1-\mu) y \|^{2} \\
= & \|x\|^{2}\left[\lambda^{2} \mu(1-\mu)+\lambda(1-\lambda) \mu^{2}\right] \\
& \quad+\|y\|^{2}\left[(1-\lambda)^{2} \mu(1-\mu)+\lambda(1-\lambda)(1-\mu)^{2}\right] \\
= & {[\lambda+\mu-2 \lambda \mu]\left[\lambda \mu\|x\|^{2}+(1-\lambda)(1-\mu)\|y\|^{2}\right] . }
\end{aligned}
$$

In an inner product space (3) holds for all $\lambda, \mu, x$, and $y$. It implies each of the conditions $(4, \sim)$, where as before $\sim$ stands for one of the relations $=$, $\geqq$, or $\leqq$.

For each $x$ and $y$ in $E$ there exist real $\lambda$ and $\mu$, dependent on $(4, \sim) \quad x$ and $y$, such that $0<\lambda<1,0<\mu<1$, and $[\lambda+\mu-2 \lambda \mu]\left[\lambda \mu\|x\|^{2}+(1-\lambda)(1-\mu)\|y\|^{2}\right]$ $\sim \mu(1-\mu)\|\lambda x+(1-\lambda) y\|^{2}+\lambda(1-\lambda)\|\mu x-(1-\mu) y\|^{2}$. 
Cutting the freedom of $x$ and $y$ again gives still weaker conditions: For each pair $x, y$ of points of $E$ with $\|x\|=\|y\|=1$ there $(5, \sim)$ exist $\lambda$ and $\mu$ such that $0<\lambda<1,0<\mu<1$, and $(\lambda+\mu-2 \lambda \mu)(\lambda \mu+(1-\lambda)(1-\mu))$ $\sim \mu(1-\mu)\|\lambda x+(1-\lambda) y\|^{2}+\lambda(1-\lambda) \cdot\|\mu x-(1-\mu) y\|^{2}$.

Observe that most of the earlier conditions trivially imply one or more of the conditions $(5, \sim)$. $(\mathrm{JN})$ is a specialization of $(1,=)$; $(\mathrm{K})$ is a specialization of $(1, \geqq) .(1, \sim)$ is a specialization of $(2, \sim)$ and of $(4, \sim$ ) (using $\mu=1 / 2)$. $(D)$ is a specialization of $(2,=)$; $(2, \sim)$ is the special case $\mu=1 / 2$ of $(5, \sim)$. For each $i=1,2,4$, or 5 the condition $(i,=)$ is a specialization of $(i, \geqq)$ and of $(i, \leqq)$.

The simplest common generalization of both (D) and (K) is $(2, \geqq)$. That this condition is sufficient to make a normed linear space an inner-product space follows trivially from the fact that $(2, \sim)$ implies $(5, \sim)$ and

Theorem 1. Each of the three conditions $(5, \sim)$ where, $\sim$ is $=$, , or $\leqq$, is sufficient that a normed linear space be an inner-product space.

As in the proofs of Theorems 2.1 and 4.1 of my earlier paper [3] The Jordan-von Neumann theorem shows that it suffices to consider any two-dimensional subspace $\Pi$ of $E$ and to show that $C$, the set of points of norm one in $\Pi$, is an ellipse. To prove that this is true when $(5, \geqq)$ holds in $E$ let $C^{\prime}$ be the inscribed ellipse of maximal area in $C$ and let $|\cdots|$ be the norm in $\prod$ for which $C^{\prime}$ is the unit sphere. Then (see [3], the dual of Lemma 2.4) $C^{\prime}$ touches 0 in at least two pairs of points $\pm p, \pm q$, with $p \neq \pm q$.

Clearly the set $K$ of points where $C^{\prime}$ touches $C$ is a closed subset of $C$ and of $C^{\prime}$. Therefore $L$, the complemenent of $K$ in $C^{\prime}$, is open in $C^{\prime}$.

If $L$ is not empty, let $A$ be an open arc in $L$ with end points $x$ and $y$ both in $K$. Then $\prod$ is an inner-product space under $|\cdots|$ so (3) holds for $|\cdots|$. Since $C^{\prime}$ is nowhere outside $C,\|z\| \leqq|z|$ for every $z$ in $\Pi$. For the appropriate $\lambda$ and $\mu$ determined from $x$ and $y$ by $(5, \geqq)$ we have

$$
\begin{aligned}
(\lambda+\mu- & 2 \lambda \mu)(\lambda \mu+(1-\lambda)(1-\mu)) \\
& =\mu(1-\mu)|\lambda x+(1-\lambda) y|^{2}+\lambda(1-\lambda)|\mu x-(1-\mu) y|^{2} \\
& \geqq \mu(1-\mu)\|\lambda x+(1-\lambda) y\|^{2}+\lambda(1-\lambda)\|\mu x-(1-\mu) y\|^{2} \\
& \geqq(\lambda+\mu-2 \lambda \mu)(\lambda \mu+(1-\lambda)(1-\mu)) .
\end{aligned}
$$

Since the ends of the chain are equal, equality must hold all the way along the line. Since none of the numbers $\lambda, \mu, 1-\lambda, 1-\mu$ is zero, it follows that 


$$
|\lambda x+(1-\lambda) y|=\|\lambda x+(1-\lambda) y\|
$$

(and also that

$$
|\mu x-(1-\mu) y|=\|\mu x-(1-\mu) y\|) .
$$

But the vector $\lambda x+(1-\lambda) y$ can not be zero, since $x$ is not $\pm y$, so $(\lambda x+(1-\lambda) y) /\|\lambda x+(1-\lambda) y\|$ belongs to $A$ and also to $K$. This contradiction shows that $L$ must be empty; that is, that $C$ and $C^{\prime}$ coincide; that is, that $|z|=\|z\|$ for all $z$ in $\prod$. The Jordan-von Neumann theorem now asserts that $E$ is an inner-product space.

To prove the corresponding result starting from $(5, \leqq)$ it suffices to adapt this argument, replacing $C^{\prime}$ by $C^{\prime \prime}$, the ellipse of minimal area circumscribed about $C$.

Certain special cases of the conditions $(4, \sim)$ and $(5, \sim)$ are worth recording. We have already noted that $(1, \sim)$ and $(2, \sim)$ are the special cases of $(4, \sim)$ and $(5, \sim)$, respectively, for which $\mu=1 / 2$. The next conditions come from choosing $\lambda=\mu$ or $\lambda=1-\mu$ in $(4, \sim)$ and $(5, \sim)$; hence they too are criteria for a linear normed space to be an inner-product space.

For each pair $x, y$ of points of $E$ there exists $\lambda$ such that

$0<\lambda<1$ and

$$
\begin{aligned}
2\left[\lambda^{2}\|x\|^{2}+(1-\lambda)^{2}\|y\|^{2}\right] & \\
& \sim\|\lambda x+(1-\lambda) y\|^{2}+\|\lambda x-(1-\lambda) y\|^{2} .
\end{aligned}
$$

For each pair $x, y$ of points of $E$ there exists $\lambda$ such that $(7, \sim) \begin{aligned} & 0<\lambda<1 \text { and } \\ & {[1-2 \lambda(1-\lambda)]}\end{aligned}$

$$
\sim\|x\|_{\lambda x+(1-\lambda) y\left\|^{2}+\right\|(1-\lambda) x-\lambda y \|^{2} .}
$$

Note that $(6,=)$ and $(7,=)$ reduce to $(\mathrm{JN})$ when $\lambda=1 / 2$.

For each $x, y$ in $E$ such that $\|x\|=\|y\|=1$ there exists $\lambda$ such $(8, \sim)$ that $0<\lambda<1$ and $2\left[\lambda^{2}+(1-\lambda)^{2}\right]$

$$
\sim\|\lambda x+(1-\lambda) y\|^{2}+\|\lambda x-(1-\lambda) y\|^{2} .
$$

For each $x, y$ in $E$ such that $\|x\|=\|y\|=1$ there exists $\lambda$ such $(9, \sim) \quad \begin{aligned} & \text { that } 0<\lambda<1 \text { and } \\ & 2(1-2 \lambda(1-\lambda))\end{aligned}$

$$
\sim\|\lambda x+(1-\lambda) y\|^{2}+\|(1-\lambda) x-\lambda y\|^{2} .
$$

Note that $(8,=)$ and $(9,=)$ specialize to $(D)$ when $\lambda=1 / 2$.

3. Metric spaces. Throughout this section we shall be concerned with metric spaces and with properties of some such spaces which can be defined in metric terms. 
If $p$ and $r$ are points of a metric space $E$, a point $q$ of $E$ is called metrically between $p$ and $r$ whenever $p q+q r=p r$. If $\lambda$ is a number between 0 and 1 , then $q$ will be called a $\lambda$-point between $p$ and $r$ if $q$ is between $p$ and $r$ and if also $p q=\lambda p r$ (so $q r=(1-\lambda) p$ ).

It is the purpose of this section to prove a generalization of Blumenthal's results. His weaker condition, the (e4pp-2), asserts in the terms above that for each pair $p, r$ of points of $E$ and each 1/2point $q$ between $p$ and $r$ and each $s$ in $E$ the quadruple $p, q, r, s$ is isometrically embeddable in the euclidean plane. The condition ( $\mathrm{JN}$ ) is not immediately equivalent to (e4pp-2) until it is proved that the $1 / 2$-point $(p+r) / 2$ is the only $1 / 2$-point between $p$ and $r$. The direct translation of $(\mathrm{JN})$ setting $p-s=x, r-s=y$, and taking $q$ to be that $1 / 2$-point $(p+r) / 2=(x+y) / 2$, is

For each pair $p, r$ of points of $E$ there is a 1/2-point $q$ (e4pp-2.1) between $p$ and $r$ such that for each $s$ in $E$ the quadruple $p, q, r, s$ is isometrically embeddable in the euclidean plane.

A still weaker condition is obtained by translating $(1,=)$ into metric terms. This yields what shall here be called the queasy euclidean fourpoint property

For each pair $p, r$ of points of $E$ there is a $q$, not $p$ or $r$, (e4pp-3) between $p$ and $r$ such that for each $s$ in $E$ the quadruple $p, q, r, s$ is isometrically embeddable in a euclidean plane.

Let us repeat some definitions from [1]. A metric space $E$ is called metrically convex if for each $p, r$ in $E$ there is a point $q$ between $p$ and $r$. $E$ is called externally convex if for each $p$ and $r$ in $E$ there exists $q^{\prime}$ in $E$ such that $r$ is a point between $p$ and $q^{\prime} . E$ is complete if every Cauchy sequence has a limit in $E$.

Obviously all the (e4pp-i) are properties of any inner-product space. Moreover, if $i \leqq j$, then (e4pp-i) trivially implies (e4pp-j), $i, j,=0,1,2,2.1$, or 3, except that (e4pp-2) implies (e4pp-2.1) if and only if $E$ is metrically convex. Blumenthal has already proved in [2] that (e4pp-2) is sufficient that a complete, metrically convex, externally convex metric space be isometric with an inner-product space. We extend this by proving the following result:

Theorem 2. In a complete metric space E the queasy (e4pp-3) implies the feeble (e4pp-2) euclidean four-point property, and also implies metric convexity of $E$.

(a) If $q$ is the point associated with $p$ and $r$ by (e4pp-3) and if $p q$ $=\lambda p r$, then $q$ is the only $\lambda$-point between $p$ and $r$. 
If $q^{\prime}$ is any point between $p$ and $r$, define $Q^{\prime}=f\left(q^{\prime}\right)$ to be the number $p q^{\prime}$. Then $P$ is the number zero and $R$ is the number $p r$. If $s$ is also a $\lambda$-point between $p$ and $r$, then the (e4pp-3) asserts that both $q$ and $s$ are mapped by $f$ onto $p q$; that is $Q=S$. Since $Q S=0, q s=0$, or $q=s$.

Next define $K=K(p, r)$ to be the set of all points $q$ between $p$ and $r$ such that for each $s$ the quadruple $p, q, r, s$ is isometrically embeddable in a euclidean plane. Then (a) implies that

(b) $f$ is one-to-one from $K$ into the interval $[P, R]$.

Since the metric in $E$ is continuous and the equations determining whether or not a point $q$ is in $K$ are simple isometries, we have

(c) $K$ is a closed subset of $E$.

Let $\Gamma$ be the set of subsets $C$ of $K$ such that the function $f$ defined before (b) is an isometry of $C$ into $[P, R]$.

(d) If $E$ is metric, if $C \in \Gamma$, and if $C_{1}$ is the closure of $C$, then $C_{1} \in \Gamma$. If $E$ is complete, $f\left(C_{1}\right)$ is the closure of $f(C)$ in $[P, R]$.

The first result follows from completeness of the intervals $[P, R]$ and the fact that an isometry preserves Cauchy sequences. The second part follows in the same way once the completeness hypothesis makes it possible to interchange the roles of $K$ and $[P, R]$.

We shall soon bend our efforts to showing that there is a maximal element of $\Gamma$, but first we show that certain kinds of elements of $\Gamma$ are not maximal there. (d) already implies:

$\left(\mathrm{d}^{\prime}\right)$ If $E$ is complete and $C \in \Gamma$ and $f(C)$ is not closed, then $C$ is not maximal in $\Gamma$.

For then $C$ is not closed either, and therefore is a proper subset of its closure.

We need a well-known auxiliary result.

(e) If $p p^{\prime}+p^{\prime} r^{\prime}+r^{\prime} r=p r$ (so $p^{\prime}$ and $r^{\prime}$ are between $p$ and $r$ ), then every point $q^{\prime}$ between $p^{\prime}$ and $r^{\prime}$ is also between $p$ and $r$.

$p^{\prime} r^{\prime}=p^{\prime} q^{\prime}+q^{\prime} r^{\prime}$ implies that $p p^{\prime}+p^{\prime} q^{\prime}+q^{\prime} r^{\prime}+r^{\prime} r=p r$, so $p r \leqq p q^{\prime}$ $+q^{\prime} r \leqq p p^{\prime}+p^{\prime} q^{\prime}+q^{\prime} r^{\prime}+r^{\prime} r=p r$. Hence $=$ holds through the whole chain; the first such equality says that $q^{\prime}$ is between $p$ and $r$.

(f) If $C \in \Gamma$ and if $f(C)$ is closed in $[P, R]$ but is not all of $[P, R]$, then $C$ is not maximal in $\Gamma$; that is, there is a $C^{\prime}$ in $\Gamma$ such that $C^{\prime}$ properly contains $C$.

If $f(C)$ is closed but is not all of $[P, R]$, let $\left[P^{\prime}, R^{\prime}\right]$ be a subinterval of $[P, R]$ such that $P^{\prime}$ and $R^{\prime}$ are in $f(C)$ but the open inerval $\left(P^{\prime}, R^{\prime}\right)$ is in the complement of $f(C)$. Then apply (e4pp-3) to $p^{\prime}=f^{-1}\left(P^{\prime}\right)$ and $r^{\prime}=f^{-1}\left(R^{\prime}\right)$ to get a point $q^{\prime}$. By (e) $q^{\prime}$ is between $p$ and $r$.

$f$ is an isometry of $C$ into $[P, R]$; if $s$ is a point of $E$, take a plane II containing the line through $P$ and $R$ and take a point $S=f(s)$ in $\prod$ 
so that $P S=p s$ and $P R=p r$. (The triangle inequality asserts that this is always possible.) That $C \subseteq K$ means that $f$ is an isometry of $C \cup(s)$ into $\prod$. Let $Q^{\prime}=f\left(q^{\prime}\right)$ and let $C^{\prime}=C \cup\left(q^{\prime}\right)$. By the (e4pp-3) applied to $p^{\prime}, q^{\prime}, r^{\prime}$, and $s$ we have $q^{\prime} s=Q^{\prime} S$. Hence $Q^{\prime} \in K$ and $f$ is an isometry of $C^{\prime} \cup(s)$ into $\Pi$. This shows that $C^{\prime} \in \Gamma$. $Q^{\prime} \in A$ so $q^{\prime} \notin C$ and $C^{\prime} \neq C$.

(g) If $\Gamma$ is ordered by $\supseteq$, there is a maximal element $A$ of $\Gamma$.

Let $\Gamma^{\prime}$ be any simply ordered subsystem of the partially ordered system $\Gamma$, and let $C$ be the union of all the sets $C^{\prime}$ in $\Gamma^{\prime}$. Then $f$ is an isometry on $C$ also, and $C \subseteq K$; hence $C \in \Gamma$. By the Hausdorff maximum principle (see, for example, Kelley, General topology, p. 31) there is a maximal $C$ in $\Gamma$.

(h) If $E$ is complete, then $K$ is an arc connecting $p$ to $r, f$ is an isometry of $K$ with all of $[P, R]$, and all points of $E$ between $p$ and $r$ are points of $K$.

If $A$ is a maximal element of $\Gamma$, by $\left(\mathrm{d}^{\prime}\right) f(A)$ is closed in $[P, R]$. By (f) $[P, R]=f(A) \subseteq f(K) \subseteq[P, R]$. By (b) $K=A$. Since $f$ is an isometry between $K$ and $[P, R], K$ is an arc between $p=f^{-1}(P)$ and $r=f^{-1}(R)$. (a) implies that every point between $p$ and $r$ is on $K$.

This completes the proof of Theorem 2 when it is observed that each point of the arc $K$ is a point between $p$ and $r$, so $E$ is metrically convex.

Corollary 1. Let E be a complete, externally convex metric space. $E$ satisfies the (e4pp-3) if and only if it is isometric to an inner-product space.

The midpoint of $K$ satisfies (e4pp-2.1) and is the only 1/2-point between $p$ and $r$ by (a) (or (h)); hence $E$ satisfies the (e4pp-2), and is metrically convex. By Blumenthal's theorem $E$ is isometric to an inner-product space.

Note that Blumenthal's example of a convexly metrized tripod shows that the condition $q s=Q S$ throughout this argument can not be replaced by $Q S \geqq q s$, so a direct translation of $(1, \geqq)$ or of $(\mathrm{K})$ or even of (K) with $a=1 / 2$ (which could best be located in our symbolism as $(\mathrm{JN}, \geqq)$ ) is not sufficient to characterize inner-product spaces among complete, metrically convex, externally convex metric spaces.

Note also that while our condition (e4pp-3) may hold in a metric space, it is not immediately obvious that the same condition need hold in the completion, the catch being that as $p_{n} \rightarrow p$ and $r_{n} \rightarrow r$ the appropriate $\lambda_{n}$ may tend to 0 or 1 . Hence on the basis of Theorem 2 we can only state the following corollary. 
Corollary 2. A complete linear metric space with (e4pp-3) is an inner-product space.

Note, however, that completeness is used in $E$ only to show that $f(C)$ is closed if $C$ is closed. In a linear metric space the condition that $q$ be chosen on the line segment between $p$ and $r$ is so strong that as we saw in $\S 2$, any one of the conditions $(5, \sim)$ suffices to determine an inner-product.

But in a linear normed space with the (e4pp-3) the condition (a) which it implies shows that the point $(1-\lambda) p+\lambda r$ satisfies $(1,=)$. Since this is a specialization of $(5,=)$, Theorem 1 implies:

Theorem 2 '. In a normed linear space $E$, (e4pp-3) implies $(1,=)$, so $E$ is an inner-product space.

\section{BiBLIOGRAPHY}

1. L. M. Blumenthal, Theory and applications of distance geometry, Oxford, 1953.

2. - Extension of a theorem of Jordan and von Neumann, Pacific J. Math. vol. 5 (1955) pp. 161-167.

3. M. M. Day, Some characterizations of inner-product spaces, Trans. Amer. Math. Soc. vol. 62 (1947) pp. 320-327.

4. P. Jordan and J. von Neumann, On inner products in normed linear spaces, Ann. of Math. vol. 36 (1935) pp. 705-718.

5. S. Kakutani, Some characterizations of Euclidean space, Jap. J. Math. vol. 16 (1939) pp. 93-97.

6. S. Kasahara, A characterization of Hilbert space, Proc. Japan. Acad. vol. 30 (1954) pp. 846-848.

7. I. J. Schoenberg, A remark on M. M. Day's characterization of Hilbert space, Proc. Amer. Math. Soc. vol. 3 (1952) pp. 961-964.

8. W. A. Wilson, $A$ relation between metric and euclidean spaces, Amer. J. Math. vol. 54 (1932) pp. 505-517.

UNIVERSITY OF ILLINOIS 